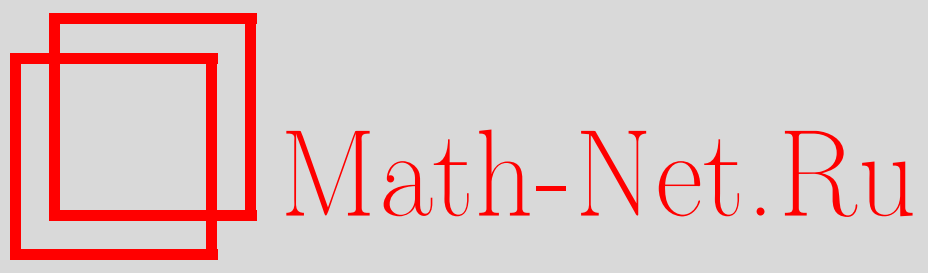

С. Г. Гиндикин, Орисферическое преобразование на римановых симметрических многообразиях некомпактного типа, Функи. анализ и его прил., 2008, том 42, выпуск 4, 50-59

DOI: https://doi.org/10.4213/faa2931

Использование Общероссийского математического портала MathNet.Ru подразумевает, что вы прочитали и согласны с пользовательским соглашением

http://www . mathnet.ru/rus/agreement

Параметры загрузки:

IP : 54.237 .59 .107

26 апреля 2023 г., 12:26:28

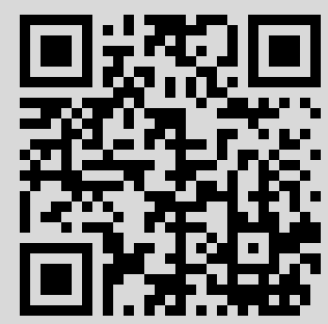




\title{
Орисферическое преобразование на римановых симметрических многообразиях некомпактного типа
}

\author{
(c) 2008. С. Г. Гиндикин
}

\section{Израилю Моисеевичу Гельфанду в связи с его 95-летием}

Мы обсудим в этой статье один из самых замечательных проектов И. М. Гельфанда - интегральную геометрию [2]. Этому проекту около 50 лет. Я был свидетелем его первой презентации, и значительная часть моей математической жизни была связана с интегральной геометрией. Это был огромный далеко идущий план перестроить гармонический анализ на полупростых группах Ли на базе интегральных преобразований типа преобразования Радона и превратить гармонический анализ в более широкую область геометрического анализа, оперирующую с геометрическими структурами, уже не связанными обязательно с группами.

Эта новая область началась в 1959 г. с фундаментальной статьи [3] И. М. Гельфанда и М. И. Граева, но она имела замечательную предысторию. Оказалось, что большая часть доказательства И. М. Гельфанда и М. А. Наймарка аналога формулы Планшереля для группы Лоренца в 1947 г. была посвящена восстановлению функций в $\mathbb{C}^{3}$ через их интегралы по прямым, пересекающим гиперболу [7]. Этой геометрической интерпретации не было в статье, и, вероятно, ее осознание послужило импульсом к статье [3].

Основное наблюдение в этой статье [3] состояло в том, что для некоторых однородных многообразий (конкретно, для комплексных полупростых групп Ли и соответствующих римановых симметрических многообразий) имеется замечательное интегральное преобразование типа преобразования Радона - орисферическое преобразование. В этом преобразовании функции на однородном многообразии интегрируются по орисферам - орбитам максимальных унипотентных подгрупп. Идея работать с орисферами, а не с геодезическими подмногообразиями, принципиальна и восходит к наблюдению в геометрии Лобачевского, что орисферы геометрически часто важнее гиперплоскостей (оба объекта отвечают двум характеристическим свойствам евклидовых гиперплоскостей быть вполне геодезическими подмногообразиями и быть сферами бесконечного радиуса, - которые расщепляются в неевклидовом случае).

Орисферическое преобразование является двойником аналога преобразования Фурье на симметрическом многообразии: они связаны коммутативным преобразованием Меллина размерности, равной рангу. (Напомним, что в евклидовом случае преобразования Радона и Фурье связаны одномерным преобразованием Фурье). Эта связь дает возможность найти явную формулу обращения для орисферического преобразования, пользуясь формулой Планшереля для комплексных полупростых групп Ли. Она имеет замечательное сходство с формулой обращения Радона в нечетной размерности. А именно, некоторый дифференциальный оператор применяется к орисферическому преобразованию вдоль 
семейства параллельных орисфер и усреднение по множеству орисфер, проходящих через точку, реконструирует функцию в этой точке. Автоматически в реконструкции значения в точке участвуют только интегралы по орисферам, близким к точке. Формулы обращения в интегральной геометрии, которые имеют такую структуру с усреднением результата применения дифференциального оператора, называются локальными.

Существование такой геометризации спектрального анализа для некоторых однородных многообразий не могло быть случайным. Основная цель проекта состояла в развитии прямых методов геометрического анализа для обращения подобного рода интегральных преобразований типа преобразования Радона, без использования действия групп. Хотя в [3] обращение орисферического преобразования для комплексных групп было следствием формулы Планшереля, в будущем процесс должен был стать обратным и формула Планшереля на симметрических и более общих однородных многообразиях должна была получаться с помощью обращения орисферического преобразования. Прошло 10 лет, прежде чем такие прямые методы стали появляться.

В [5] было найдено замечательное средство строить обращение для группы $G=G L(n ; \mathbb{C})$ - оператор $\kappa$. Исходное наблюдение состояло в том, что орисферы в этом случае можно рассматривать как некоторые $k$-мерные плоскости, $k=n(n-1) / 2$, в $\mathbb{C}^{N} \supset G, N=n^{2}$. Был развит общий метод реконструкции функций через их интегралы по $k$-мерным плоскостям из некоторого $N$-параметрического семейства, без всякой связи с группой $G$. В частном случае это дает обращение орисферического преобразования для $G L(n ; \mathbb{C})[4] . \mathrm{B}$ результате это дает новый метод получения формулы Планшереля для этой группы. История усовершенствования доказательства этой формулы была длинной: от исключительно сложного доказательства Гельфанда и Наймарка в 1950 г. до очень изящного доказательства Гельфанда и Граева на основе интеграла М. Рисса в 1956 г. Новое доказательство было особенно просто и одновременно возвращало к доказательству Гельфанда и Наймарка 1947 г. для $n=2$, которое мы обсуждали выше и которое авторы тогда не смогли обобщить на произвольное $n$. Для других комплексных полупростых групп Ли орисферы не могут быть сделаны плоскими, и в [9] было предложено обобщение метода оператора $\kappa$ на кривой случай, достаточное для обращения орисферического преобразования для всех комплексных полупростых групп Ли. Еще остаются важные проблемы, связанные с локальными формулами обращения на некоторых псевдоримановых симметрических многообразиях, однако не вызывает сомнений, что локальные формулы обращения на симметрических пространствах могут быть получены прямыми аналитическими методами.

Для общих римановых симметрических многообразий локальных формул обращения орисферического преобразования нет. Простейшим примером являются четномерные пространства Лобачевского (ср. с формулой обращения Радона в четномерном пространстве). Локальная формула обращения существует тогда и только тогда, когда все кратности корней четны. В общем случае формулы обращения были найдены в [12], [1] (см. детали ниже). Эта формула содержит усреднение оператора, действующего вдоль семейств параллельных орисфер, который является композицией локального (дифференциального) оператора (не отличающегося от оператора в локальных формулах обращения) и нелокального сингулярного интегрального оператора. Последний существенно зависит от типа системы корней и включает некоторые новые подсистемы простых корней. 
Они кажутся очень интересными. Но эта комбинаторная красота порождает сомнения в том, что такие формулы можно получить прямыми аналитическими методами (как это было в локальном случае). Мы даем в этой статье модификацию нелокального оператора, в которой он универсально выражается через простые корни, и это делает реалистичным прямой аналитический путь без серьезного использования теории представлений.

С самого начала идея сделать орисферическое преобразование основным средством теории представлений также и в случае вещественных групп встретила серьезное препятствие: оно имеет нетривиальное ядро во многих случаях, начиная с группы $S L(2 ; \mathbb{R})$. Более точно, дискретные серии лежат в ядре орисферического преобразования, и вообще это преобразование связано только с максимально непрерывным спектром. Это драматически ограничивает область применения интегральной геометрии в теории представлений и явилось, вероятно, причиной, по которой многие специалисты были пессимистично настроены относительно перспектив интегральной геометрии в гармоническом анализе на вещественных группах и, более общо, на псевдоримановых симметрических многообразиях. Я хочу подчеркнуть, что И. М. Гельфанд всегда верил в универсальные возможности метода орисфер, который, после подходящих модификаций, должен охватывать и дискретные серии. Интересный эксперимент в этом направлении был сделан в [6]: если в трехмерном мнимом пространстве Лобачевского добавить к интегралам по орисферам интегралы по некоторым вырожденным орициклам, то ядро исчезает. К сожалению, эта конструкция специфична для этого примера и не работает уже для мнимого пространства Лобачевского большей размерности. В [10] я предложил некоторую модификацию орисферического преобразования - орисферическое преобразование Коши. Идея состоит в том, что если имеется недостаточно вещественных орисфер, то надо добавить какие-то комплексные, заменив интегрирование по вещественным орисферам сверткой с ядром типа ядра Коши с особенностями на этих комплексных орисферах. Другими словами, $\delta$-функция заменяется на ядро Коши. Это работает для $S L(2 ; \mathbb{R})$ и для всех голоморфных дискретных серий. Я верю, что после подходящих модификаций это должно работать для всех псевдоримановых симметрических многообразий и что исходный план Гельфанда можно реализовать.

Орисферическое преобразование на римановых симметрических многообразиях некомпактного типа. Начнем детальное обсуждение этого преобразования и его обращения. Мы упоминали выше, что явная формула для обращения известна в принципе, но ее структура нуждается в уточнении. Начнем с основных определений. Мы рассматриваем однородное многообразие $X=G / K$, где $G$ - связная полупростая группа Ли с конечным центром, а $K-$ ее максимальная компактная подгруппа. Пусть

$$
G=K A N
$$

- разложение Ивасавы, $A$ - картановская подгруппа симметрического многообразия $X$ и $N$ - максимальная унипотентная подгруппа. Пусть $\Sigma, \Sigma_{+}$ - ограниченные системы корней и соответственно положительных корней, а $\Pi \subset \Sigma_{+}$- система простых корней. Пусть, далее, $M$ - централизатор подгруппы $A$ в $K$. Назовем $\Xi=G / M N$ орисферическим многообразием. Оно имеет ту же размерность, что и $X$. При этом $P=M A N$ - параболическая подгруппа и $F=G / P=K / M-$ флаговое многообразие. Имеется каноническое расслоение $\Xi \rightarrow F$ со слоем $A$. 
Рассмотрим двойное расслоение

$$
X \leftarrow G / M \rightarrow \Xi,
$$

индуцированное разложением Ивасавы. Точки $\xi \in \Xi$ отвечают некоторым подмногообразиям $E(\xi)$ коразмерности, равной размерности $l$ группы $A$ (т. е. рангу многообразия $X)$, - орисферам. Они являются орбитами подгруппы $N$ и всех сопряженных подгрупп. Если фиксировать точку $x_{0} \in X$, то параметры орисфер, проходящих через эту точку, образуют сечение $S\left(x_{0}\right)$ расслоения $\Xi \rightarrow F$. Это дает тривиализацию расслоения: $\Xi=F \times A$, и мы параметризуем орисферы. Если $u_{0} \in F=K / M$ отвечает орисфере $\left\{x_{0} N\right\}$, то $E(u, a)=\left\{x_{0} N a k(u)\right\}, u \in F$, $k(u) \in K, k(u) u_{0}=u, a \in A$. На $\Xi$ действует $A$ (вдоль слоев расслоения), и это действие коммутирует с действием группы $G$. Возникает расширение группы автоморфизмов многообразия $\Xi$ до $G \times A$, и это расширение исключительно важно для гармонического анализа на $\Xi$.

Фиксируем инвариантные меры $d a, d n$ на $A, N$ соответственно. Тогда $d x=$ $a^{-2 \rho} d a d n, x=x_{0} a n,-$ инвариантная мера на $X ; a^{-2 \rho}=\exp (-2 \rho(\ln a)), 2 \rho-$ сумма корней в $\Sigma_{+}$(с их кратностями). Меры $a^{-2 \rho} d n$ на $E(u, a)$ согласованы с действием группы $G$ на $\Xi$. Мы определяем орисферическое преобразование функций $f \in C_{0}^{\infty}(X)$ как

$$
\hat{f}(u, a)=a^{-2 \rho} \int_{E(u, a)} f d n=a^{-2 \rho} \int_{N} f\left(x_{0} n a k(u)\right) d n .
$$

Это сплетающий оператор между функциями на $X$ и на $\Xi$ (он не зависит от тривиализации расслоения $\Xi \rightarrow F$, ассоциированной с фиксированной точкой $\left.x_{0}\right)$.

Мы рассматриваем преобразование Меллина относительно действия подгруппы $A$ на слои расслоения $\Xi \rightarrow F$ для функций $\varphi(u, a)$ на $\Xi$ :

$$
\mathscr{M} \varphi(u, r)=\int_{A} \varphi(u, a) \exp ((\rho+i r)(\ln a)) d a, \quad r \in \mathfrak{a}^{*},
$$

где $\mathfrak{a}^{*}$ - это дуальное пространство к алгебре Ли a подгруппы $A$. Для фиксированного $r$ имеется индуцированное действие группы $G$, и это представление основной серии. Назовем композицию этих двух интегральных преобразований преобразованием Фурье:

$$
\begin{aligned}
\tilde{f}(u, r)=\mathscr{M}(\hat{f})(u, r) & =\int_{A} \hat{f}(u, a) \exp ((\rho+i r)(\ln a)) d a \\
& =\int_{A} f\left(x_{0} n a k(u) \exp ((-\rho+i r)(\ln a)) d n d a, \quad r \in \mathfrak{a}^{*} .\right.
\end{aligned}
$$

Формула Планшереля дает обращение этого преобразования Фурье:

$$
f\left(x_{0}\right)=\int_{\mathfrak{a}^{*} \times F} \tilde{f}(u, r) p(r) d r d u,
$$

где $d u$ - это инвариантная мера на $F$ (относительно действия $K$ ) и $p(r)$ называется плотностью меры Планшереля. Хариш-Чандра выразил $p$ через специальную функцию $c(r)$, которая называется теперь $c$-функцией Хариш-Чандры:

$$
p(r)=\frac{1}{|c(r)|^{2}},
$$

и дал интегральную формулу для $c$-функции. 
В [11] была найдена точная формула для $c$-функции. Фиксируем картановское скалярное произведение $\langle r, s\rangle$ на $\mathfrak{a}$ и отождествим $\mathfrak{a}^{*}$ с $\mathfrak{a}$. Тогда

$$
p(r)=c \frac{1}{\prod_{\alpha \in \Sigma_{+}}\left|B\left(\frac{m_{\alpha}}{2}, \frac{m_{\alpha / 2}}{4}+i \frac{\langle\alpha, r\rangle}{\langle\alpha, \alpha\rangle}\right)\right|^{2}},
$$

где $m_{\alpha}$ - кратность корня $\alpha$, а $B$ - бета-функция Эйлера. Здесь и далее мы обозначаем через $c$ нормализующие константы.

Обращение орисферического преобразования. Идея работы [12] состояла в том, чтобы построить обращение орисферического преобразования $\hat{f}(u, a) \rightarrow f(x)$, пользуясь явной формулой Планшереля, как это было сделано в [3] для комплексных групп. Поскольку орисферическое преобразование является сплетающим оператором относительно действия $K$, для реконструкции в точке $x_{0}$ достаточно рассматривать сферически симметричные функции $f$ относительно этой точки: $f(x)=\varphi(a), a \in A$. Тогда $a^{\rho} \hat{f}(u, a)$ зависит только от $A$, и эта функция будет симметричной относительно группы Вейля: $a^{\rho} \hat{f}(u, a)=\check{\varphi}(s)$, $s=\ln a, s \in \mathfrak{a}$. Аналогично, $\tilde{f}(u, r)$ зависит только от $r \in \mathfrak{a}^{*}=\mathfrak{a}$. Эта функция $\tilde{\varphi}(r)$ является (евклидовым) преобразованием Фурье функции $\check{\varphi}(s)$, и она инвариантна относительно группы Вейля. В результате

$$
f\left(x_{0}\right)=\varphi(0)=(q * \check{\varphi})(0), \quad \text { где } p(r)=\tilde{q}(r) .
$$

Таким образом, $q(s)$ - это обратное преобразование Фурье плотности меры Планшереля $p(r)$ в обычном смысле. Проблема заключается в том, что надо вычислить преобразование Фурье произведения большого числа $B$-функций.

Первый шаг состоит в отделении полиномиального множителя, который после преобразования Фурье переходит в дифференциальный оператор. В произведении по $\Sigma_{+}$множители, отвечающие корням $\alpha$ с четными кратностями, являются полиномами. Поэтому для вычисления неполиномиального множителя мы должны рассматривать лишь систему $\Sigma_{+}^{\text {odd }}$ корней с нечетными кратностями.

Лемма 1. Мы имеем

$$
p(r)=p_{l}(r) p_{n}(r)
$$

где $p_{l}(r)$ - это полином, а

$$
p_{n}(r)=\prod_{\alpha \in \Sigma_{+}^{\text {odd }}}(\cos ) \operatorname{th}\left(\pi \frac{\langle\alpha, r\rangle}{\langle\alpha, \alpha\rangle}\right) .
$$

Здесь (со)th - это cth, если $\frac{m_{\alpha / 2}}{2}$ нечетно, $и$ th в других случаях.

Доказательство. Если $\alpha / 2$ не является корнем, то мы имеем множитель

$$
\left|\frac{\Gamma\left(\frac{m_{\alpha}}{2}+i \frac{\langle\alpha, r\rangle}{\langle\alpha, \alpha\rangle}\right)}{\Gamma\left(i \frac{\langle\alpha, r\rangle}{\langle\alpha, \alpha\rangle}\right)}\right|^{2}
$$

который, после отделения полиномиального множителя, преобразуется в

$$
\left|\frac{\Gamma\left(\frac{1}{2}+i \frac{\langle\alpha, r\rangle}{\langle\alpha, \alpha\rangle}\right)}{\Gamma\left(i \frac{\langle\alpha, r\rangle}{\langle\alpha, \alpha\rangle}\right)}\right|^{2}=\operatorname{th}\left(\pi \frac{\langle\alpha, r\rangle}{\langle\alpha, \alpha\rangle}\right),
$$


поскольку

$$
|\Gamma(i x)|^{2}=\frac{\pi}{x \operatorname{sh}(\pi x)}, \quad\left|\Gamma\left(\frac{1}{2}+i x\right)\right|^{2}=\frac{\pi}{\operatorname{ch}(\pi x)} .
$$

Если $\alpha / 2$ - корень, то $m_{\alpha}$ нечетно $(1,3$ или 7$)$, и $m_{\alpha / 2}$ четно и кратно 4 , если $m_{\alpha} \neq 1$. Удобно объединить множители для $\alpha$ и $\alpha / 2$ в функцию $p_{\alpha}(r)$, соответствующую симметрическому многообразию ранга 1 , и преобразовать это выражение, пользуясь формулой удвоения $\Gamma(2 x)=2^{2 x-1} \pi^{-1 / 2} \Gamma(x) \Gamma\left(\frac{1}{2}+x\right)$, в

$$
4 \pi\left|\frac{\Gamma\left(\frac{1}{4} m_{\alpha / 2}+\frac{1}{2}+i \frac{\langle\alpha, r\rangle}{\langle\alpha, \alpha\rangle}\right) \Gamma\left(\frac{1}{4} m_{\alpha / 2}+\frac{1}{2} m_{\alpha}+i \frac{\langle\alpha, r\rangle}{\langle\alpha, \alpha\rangle}\right)}{\Gamma\left(i \frac{\langle\alpha, r\rangle}{\langle\alpha, \alpha\rangle}\right) \Gamma\left(\frac{1}{2}+i \frac{\langle\alpha, r\rangle}{\langle\alpha, \alpha\rangle}\right)}\right|^{2} .
$$

Мы опять отделяем полиномиальный множитель и, если $\frac{1}{4} m_{\alpha / 2}$ целое $\left(m_{\alpha}\right.$ всегда нечетно, если $\alpha / 2$ - корень), получаем $\operatorname{th}\left(\pi \frac{\langle\alpha, r\rangle}{\langle\alpha, \alpha\rangle}\right)$, а если не целое, то получаем обратное выражение $\operatorname{cth}\left(\pi \frac{\langle\alpha, r\rangle}{\langle\alpha, \alpha\rangle}\right)$. Заметим, что во всех случаях $\frac{1}{4} m_{\alpha / 2}+\frac{1}{2} m_{\alpha}$ - это ненулевое целое число. Таким образом мы можем уничтожить $x$ в знаменателе у $|\Gamma(i x)|^{2}$ при отделении полиномиального множителя.

Одновременно мы вычислили полиномиальный множитель $p_{l}(r)$.

Лемма 2. Имеем

$$
p_{l}(r)=c \prod_{\alpha \in \Sigma_{+}^{0}} p_{l, \alpha}(r)
$$

где $\Sigma_{+}^{0}$ - это множество таких положительных корней $\alpha$, что $2 \alpha$ не является корнем, и

$$
\begin{aligned}
& \left\{\begin{array}{lr}
\left|\frac{\Gamma\left(\frac{m_{\alpha}}{2}+i\langle\alpha, r\rangle\langle\alpha, \alpha\rangle\right)}{\Gamma(i\langle\alpha, r\rangle\langle\alpha, \alpha\rangle)}\right|^{2}, & \text { если } \alpha / 2 \text { - не корень и } m_{\alpha} \text { четно, } \\
\langle\alpha, r\rangle\left|\frac{\Gamma\left(i \frac{m_{\alpha}}{2}+\langle\alpha, r\rangle\langle\alpha, \alpha\rangle\right)}{\Gamma(i\langle\alpha, r\rangle\langle\alpha, \alpha\rangle+1 / 2)}\right|^{2}, & \text { если } \alpha / 2-\text { не корень } и m_{\alpha} \text { нечетно, }
\end{array}\right. \\
& p_{l, \alpha}=\left\{\left|\frac{\Gamma\left(\frac{1}{4} m_{\alpha / 2}+\frac{1}{2}+i \frac{\langle\alpha, r\rangle}{\langle\alpha, \alpha\rangle}\right) \Gamma\left(\frac{1}{4} m_{\alpha / 2}+\frac{1}{2} m_{\alpha}+i \frac{\langle\alpha, r\rangle}{\langle\alpha, \alpha\rangle}\right)}{\left(\Gamma\left(\frac{1}{2}+i \frac{\langle\alpha, r\rangle}{\langle\alpha, \alpha\rangle}\right)\right)^{2}}\right|^{2},\right. \\
& \text { если } \alpha / 2 \text { - корень } и \frac{m_{\alpha / 2}}{4} \text { челое, } \\
& \frac{1}{\langle\alpha, r\rangle}\left|\frac{\Gamma\left(\frac{1}{4} m_{\alpha / 2}+\frac{1}{2}+i \frac{\langle\alpha, r\rangle}{\langle\alpha, \alpha\rangle}\right) \Gamma\left(\frac{1}{4} m_{\alpha / 2}+\frac{1}{2} m_{\alpha}+i \frac{\langle\alpha, r\rangle}{\langle\alpha, \alpha\rangle}\right)}{\left(\Gamma\left(i \frac{\langle\alpha, r\rangle}{\langle\alpha, \alpha\rangle}\right)\right)^{2}}\right|^{2}, \\
& \text { если } \alpha / 2 \text { - корень и } \frac{m_{\alpha / 2}}{4} \text { не иелое, }
\end{aligned}
$$

Во всех случаях

$$
\operatorname{deg} p_{l, \alpha}=m_{\alpha}+m_{\alpha / 2} \text {. }
$$

Следующий шаг состоит в вычислении обратного преобразования Фурье функции $p_{n}(r)$. Разумеется, обратное преобразование Фурье каждого множителя хорошо известно:

$$
\operatorname{th}(\pi x)=\left(\frac{1}{2 \pi i \operatorname{sh}\left(\frac{x}{2}\right)}\right)^{\sim}, \quad \operatorname{cth}(\pi x)=\left(\frac{1}{2 \pi i} \operatorname{cth}\left(\frac{x}{2}\right)\right)^{\sim} .
$$


Если бы мы имели произведение гиперболических тангенсов от линейно независимых линейных функций от $r$, не было бы проблем с вычислением преобразования Фурье. Однако у нас произведение большого числа множителей с линейно зависимыми аргументами. В [12] мы предложили очень простой прием с применением формулы

$$
\text { th } x \operatorname{th} y \operatorname{th}(x+y)=\operatorname{th} x+\operatorname{th} y-\operatorname{th}(x+y),
$$

который дает возможность кардинально уменьшить количество множителей и, в конечном счете, получить произведение гиперболических тангенсов от линейно независимых форм. Мы экспериментально нашли такие редукции для всех классических групп. В [1] Беерендс сделал очень удачное наблюдение, что для произвольного множества $R$ линейно независимых корней

$$
c_{R} \prod_{\alpha \in \Sigma_{+}} \operatorname{th}\left(\pi \frac{\alpha}{\langle\alpha, \alpha\rangle}\right)=\sum_{s \in W} \operatorname{sign} s \prod_{\alpha \in R} \operatorname{th}\left(\pi \frac{s \alpha}{\langle\alpha, \alpha\rangle}\right) .
$$

Часто $c_{R}=0$, но в [1] было доказано, что для всех систем корней существуют подсистемы $R$ с ненулевыми коэффициентами. Таким образом, $p_{n}$ всегда можно преобразовать к выражению, для которого можно явно вычислить преобразование Фурье. Такие подсистемы не единственны и сильно различаются для разных корневых систем. Комбинаторная природа таких минимальных подсистем выглядит очень привлекательной, но, с точки зрения изложенной выше философии интегральной геометрии, было бы важно в окончательной формуле избежать ссылок на специфические корневые системы.

Прежде всего заметим, что

$$
\operatorname{cth} x=\operatorname{th}(x+i \pi / 2),
$$

и мы можем включить cth в наши аддитивные преобразования (свойство корней соответствовать cth совместимо с аддитивной структурой корней).

Предложение. Имеем

$$
2^{l} \prod_{\alpha \in \Sigma_{+}^{\text {odd }}}(\operatorname{co}) \operatorname{th}\left(\pi \frac{\alpha}{\langle\alpha, \alpha\rangle}\right)=\sum_{s \in W} \operatorname{sign} s \prod_{\alpha \in \Pi^{\text {odd }}}\left(1+\operatorname{th}\left(\pi \frac{s \alpha}{\langle\alpha, \alpha\rangle}\right)\right),
$$

где П ${ }^{\text {odd }}$ - система простых корней для $\Sigma_{+}^{\text {odd }}$ и $l$ - их число («нечетный ранг»).

Доказательство. Удобно при доказательстве таких тождеств с th воспользоваться тем фактом, что можно заменить th $x$ на $\operatorname{sgn}(x)$. Мы имеем

$$
\lim _{a \rightarrow \infty} \operatorname{th}(a x)=\operatorname{sgn}(x) .
$$

Этот предел отвечает переходу к соответствующему симметрическому пространству нулевой кривизны [8]. Мы обсудим эту связь ниже. Заметим, что $1+$ $\operatorname{sgn}(x)=2 \chi(x)$ (функция Хевисайда). Из тождества (7) следует, что тождество (8) в предложении верно с какой-то константой $c$ (вместо $2^{l}$ ), которая может оказаться нулем. Причина в том, что правая часть формулы (8) является комбинацией правых частей формул (7) для всех подсистем $R$. Обе части в $(8)$ после замены th на sgn постоянны в камерах Вейля, и каждый член в правой части равен нулю вне одной камеры. Сравнивая значения обеих частей в главной камере, мы и получаем $c=2^{l}$. В некотором смысле полученное тождество является производящим для тождеств (7) для всех $R$. 
Объединяя наши вычисления, мы получаем следующее утверждение.

Теорема. Пусть $f(u, a)=\varphi(\ln a) \in C_{0}^{\infty}(X)$ - сферически симметричная функиия и $\hat{\varphi}(r)$ - ее орисферическое преобразование. Тогда

$$
\varphi(0)=c \int_{\mathfrak{a}} \prod_{\alpha \in \Pi^{\text {odd }}}\left[(\operatorname{sh}(\langle\alpha, s\rangle)-i 0)^{-1}\{\operatorname{ch}(\langle\alpha, s\rangle)\}\right] p_{l}(D)(\exp \langle\rho, s\rangle \hat{\varphi}(s)) d s .
$$

Здесь

$$
\{\operatorname{ch}(\langle\alpha, s\rangle)\}= \begin{cases}\operatorname{ch}(\langle\alpha, s\rangle), & \text { если } \frac{m_{\alpha / 2}}{2} \text { челое } \\ 1 & \text { в остальных случаях }\end{cases}
$$

$\rho$ - это полусумма положительных корней.

Мы можем заменить здесь произведение по $\Pi^{\text {odd }}$ произведением по всем простым корням $\alpha \in \Pi \subset \Sigma_{+}^{0}$.

Дифференциальный оператор $p_{l}(D)=\prod_{\alpha \in \Sigma+^{0}} p_{l, \alpha}\left(D_{\alpha}\right)$ получается из полиномов $p_{l, \alpha}(r)$ заменой $i \frac{\langle\alpha, r\rangle}{\langle\alpha, \alpha\rangle}$ на операторы $D_{\alpha}$ дифференцирования в направлении корня $\alpha$. Мы воспользовались тем, что $1+$ th $x$ - это преобразование Фурье обобщенной функции

$$
\frac{1}{2 \pi i}(\operatorname{sh}(u / 2)-i 0)^{-1}=\frac{1}{2} \delta\left(\operatorname{sh}(u / 2)+\frac{1}{2 \pi i} \operatorname{sh}(u / 2)\right)^{-1} .
$$

Эта теорема - результат прямого вычисления преобразования Фурье после того, как мы оставили в неполиномиальной части число множителей, равное рангу, следуя доказанному выше предложению. Функция $\exp \langle\rho, s\rangle) \hat{\varphi}(s)$, так же как ее преобразование Фурье $\tilde{\varphi}(r)$ и плотность меры Планшереля $p(r)$, инвариантна относительно группы Вейля $W$. Мы преобразовали неполиномиальную часть функции $p_{n}(r)$ в сумму, все члены которой - результаты действия элементов группы Вейля $W$ на один из них. Поэтому интегрирование всех этих членов дает одинаковые результаты, и мы можем оставить в окончательной формуле только член, отвечающий $\Pi^{\text {odd }}$.

При расширении Подd до полной системы простых корней П при помощи простых корней $\alpha$ четной кратности мы можем заменять 1 на $1+\operatorname{th}\left(\pi \frac{\langle\alpha, r\rangle}{\langle\alpha, \alpha\rangle}\right)$, поскольку мы добавляем лишь некоторые функции, которые дают нули после интегрирования ввиду антисимметрии относительно подходящих элементов группы Вейля.

Замечание. Этот прием, который приводит к формуле, общей для четных и нечетных кратностей, аналогичен подобному приему для преобразования Радона. Если в стандартных обозначениях $\hat{f}(\xi, p)$ - это преобразование Радона функции $f(x), x \in \mathbb{R}^{n}$, то формула обращения локальна для нечетных $n$ и нелокальна для четных. Однако если рассмотреть

$$
\check{f}(\xi, p)=\int_{\mathbb{R}}(p-i 0)^{-1} \hat{f}(\xi, p) d p,
$$

то $f(x)$ получается усреднением $\frac{\partial^{(n-1)} \check{f}(\xi, p)}{\partial p^{(n-1)}}$ независимо от четности $n$. Преобразование Радона $\hat{f}(\xi, p)$ четно по $p$, и если формально продифференцировать $n-1$ раз по $p$ в четномерном случае, то мы получаем нечетную функцию, которая после интегрирования по $\xi$ дает нуль. Преобразование Гильберта (свертка с обобщенной функцией $p^{-1}$ ) дает четные функции от $p$ для четных $n$ (но нечетные для нечетных $n$ ), и интеграл обязательно равен нулю. Рассмотрение $\check{f}$, 
когда четность по $p$ нарушена, объединяет эти два случая и дает формулу, не зависящую от четности.

Аналогичная ситуация имеется для орисферического преобразования. Если формально применить локальный оператор, который дает обращение для четных кратностей, в случаях когда некоторые кратности нечетны, мы получаем нуль ввиду косой симметрии подынтегральной функции. Нелокальная часть обращения как раз компенсирует косую симметрию. Соответственно мы можем преобразовать формулу (9), заменив орисферическое преобразование $f \rightarrow \hat{f}$ на (сплетающий) оператор $f \rightarrow \check{f}$ из $X$ в $\Xi$ так, что обращение станет локальным независимо от четности кратностей: $\check{f}$ - результат свертки $\hat{f}$ с обобщенной функцией типа ядра Коши, ассоциированной с простыми корнями.

Орисферическое преобразование на римановых симметрических многообразиях нулевой кривизны. Мы хотим сравнить структуры формулы обращения орисферического оператора на $X$ и аналогичной формулы в плоском случае. Напомним результаты об обращении орисферического преобразования в этом случае [8]. Пусть $\mathfrak{x}$ - подпространство в алгебре Ли Lie $(G)$, в котором инволюция, выделяющая $K$, действует как -1 ; это ортогональное дополнение к Lie $(K)$. На линейном пространстве $\mathfrak{x}=\mathbb{R}^{n}$ имеется присоединенное действие компактной группы $K$, а в результате имеется транзитивное действие полупрямого произведения $G(\mathfrak{x})=K \ltimes \mathbb{R}^{n}$, которое иногда называется картановской группой движений. Назовем однородное многообразие $\mathfrak{x}$ относительно этого действия (и с инвариантной евклидовой метрикой) симметрическим многообразием нулевой кривизны. Эта геометрическая структура реализуется на касательном пространстве к $X=G / K$.

Пусть подпространство $\mathfrak{n} \subset \mathfrak{x}$ касательно к унипотентной подгруппе $N$. Назовем орисферами на $\mathfrak{x}$ все образы подпространства $\mathfrak{n}$ при преобразовании элементами группы $G(\mathfrak{x})$. Их множество $\Xi(\mathfrak{x})$ является подмногообразием в аффинном грассманиане $(n-l)$-плоскостей в $\mathbb{R}^{n}$. Имеем $\Xi(\mathfrak{x})=F \times \mathfrak{a}, F=$ $K / M, \mathfrak{a}=\operatorname{Lie}(A)$. Точки флагового многообразия $F$ параметризуют плоскости, проходящие через 0 . Если определить эти плоскости двойственными реперами $\left(u^{1}, \ldots, u^{l}\right)$, то орисферы $\mathfrak{E}(u, r), u \in F, r \in \mathfrak{a},-$ это плоскости

$$
\left(u^{j}, x\right)=r^{j}, \quad 1 \leqslant j \leqslant l .
$$

Определим орисферическое преобразование для $f \in C_{0}^{\infty}$ как интегралы по этим плоскостям:

$$
\hat{f}(u, r)=\int_{\mathfrak{E}(u, r)} f(x) d \mu
$$

по евклидовой мере. Это ограничение преобразования Радона-Джона - интегрирования по $(n-l)$-плоскостям - на $\Xi(\mathfrak{x})$.

Поскольку семейство $\Xi(\mathfrak{x})$ инвариантно относительно сдвигов, легко обратить это орисферическое преобразование при помощи преобразования Фурье. Обращение можно представить как композицию локального и нелокального операторов. Назовем орисферическим преобразованием Коши преобразование

$$
\check{f}(u, r)=\left(\prod_{\alpha \in \Pi}(\langle\alpha, r\rangle-i 0)^{-1}\right) * \hat{f}(u, r) .
$$


Тогда

$$
f(x)=\left.c \int_{F} \prod_{\alpha \in \Sigma_{+}^{0}}\left(D_{\alpha}\right)^{m_{\alpha}} \check{f}(u, r)\right|_{r=(u, x)} d u .
$$

Мы видим, что формулы обращения для $X$ (неположительная кривизна) и $\mathfrak{x}$ (нулевая кривизна) удивительно похожи. Их нелокальные части отличаются практически выбором переменных. Центральный факт в структуре их локальных частей - разложение по положительным корням. Символы для индивидуальных корней также могут быть преобразованы друг в друга, но этот важный факт заслуживает отдельного рассмотрения.

\section{ЛитЕРАТУРА}

[1] R. J. Beerends, The Fourier transform of Harish-Chandra's c-function and inversion of Abel transform, Math. Ann., 277:1 (1987), 1-23.

[2] И. М. Гельфанд, Интегральная геометрия и ее связь с теорией представлений zpynn, УМH, 15:2 (1960), 155-164.

[3] И. М. Гельфанд, М. И. Граев, Геометрия однородных пространств, представления групп в однородных пространствах и связанные вопросы интегральной геометрии, Труды ММО, 8 (1959), 321-390.

[4] И. М. Гельфанд, М. И. Граев, Комплексы $k$-мерных плоскостей в пространстве $\mathbb{C}^{n}$ и формула Планшереля для группь $G L(n, \mathbb{C})$, Докл. АН CCCP, 179 (1968), $522-525$.

[5] И. М. Гельфанд, М. И. Граев, З. Я. Шапиро, Интегралъная геометрия на $k$-мерных плоскостях, Функц. анализ и прилож., 1:1 (1967), 15-31.

[6] И. М. Гельфанд, М. И. Граев, Применение метода орисфер к спектральному анализу функиий в вещественном и мнимом пространствах Лобачевского, Труды MMO, 11 (1962), 243-308.

[7] И. М. Гельфанд, М. А. Наймарк, Унитарные представления группь Лорениа, Изв. АН СССР, сер. матем., 11:5 (1947), 411-504.

[8] С. Г. Гиндикин, Унитарные представления групп автоморфизмов римановых симметрических пространств нулевой кривизны, Функц. анализ и прилож., 1:1 (1967), 32-36.

[9] S. Gindikin, Integral geometry on symmetric manifolds, in: Algebra and Analysis (Kemerovo, 1988), Amer. Math. Soc. Transl. (2), vol. 148, 1991, 29-37.

[10] S. Gindikin, Integral geometry on $S L(2 ; \mathbb{R})$, Math. Res. Lett., 7:4, 417-432.

[11] С. Г. Гиндикин, Ф. И. Карпелевич, Мера Планшереля на римановых симметрических пространствах неположительной кривизны, Докл. АН СССР, 145 (1962), $252-255$.

[12] С. Г. Гиндикин, Ф. И. Карпелевич, Об одной задаче интегралъной геометрии, в кн.: Памяти Н. Г. Чеботарева, изд-во Казанского ун-та, Казань, 1964, 30-43.

Department of Math., Rutgers University

Поступило в редакцию e-mail: gindikin@math.rutgers.edu 23 июля 2008 г. 